\title{
Acquired Amegakaryocytic Thrombocytopenic Purpura Progressing into Aplastic Anemia
}

\author{
Jan Philipp Novotný, Birgit Köhler, Regina Max, Gerlinde Egerer \\ Department of Medicine V, University of Heidelberg, Heidelberg, Germany \\ Received August 10, 2017; Accepted December 5, 2017.
}

Key words: Acquired amegakaryocytic thrombocytopenic purpura - Pure megakaryocytic aplasia - TPO agonist - Aplastic anemia

\begin{abstract}
Acquired amegakaryocytic thrombocytopenic purpura (AATP) is a rare hematological disorder characterized by severe thrombocytopenia and a complete or near-to complete absence of megakaryocytes in the bone marrow, while granulopoiesis, as well as erythropoiesis are usually preserved. Although autoimmune mechanisms are believed to be causative, the exact underlying pathogenesis is not known. To date, only few cases have been reported and management of this disease remains controversial with immunosuppression being the treatment modality of choice in the majority of patients. In this article, we report a case of newly acquired AATP without an associated autoimmune disease, refractory to corticoids, intravenous immunoglobulin (IVIG) and second-generation TPO (thrombopoietin) agonists, which have recently been approved for the treatment of thrombocytopenia. Finally, in accordance with other reports, disease progression into aplastic anemia has occurred.
\end{abstract}

Mailing Address: Jan Philipp Novotný, MD., Department of Medicine V, University Hospital Heidelberg, 69120 Heidelberg, Germany; Phone: +496 221568 030; e-mail: JanPhilipp.Novotny@med.uni-heidelberg.de 


\section{Introduction}

Acquired amegakaryocytic thrombocytopenic purpura (AATP), also known as acquired pure megakaryocytic aplasia, is a rare hematological disorder reported in patients ranging from 2 to 89 years of age with predilection for males in the age group above 60 years (Figure 1). It can either occur isolated or be associated with systemic lupus erythematodus (SLE) (Cela et al., 2010; Ernestho-ghoud et al., 2015) and other autoimmune diseases (Hashimoto et al., 2016), as well as hematological malignancies such as non-Hodgkin lymphoma (Lugassy, 1996).

Most patients with this disease are initially diagnosed with immune thrombocytopenic purpura (ITP) and receive treatment with corticosteroids (either prednisone or dexamethasone) until a bone marrow aspirate or biopsy is performed, which reveals normal erythro- and granulopoiesis but near to complete absence of megakaryocytes (Hoffman et al., 1982), usually. Dysplastic changes consistent with myelodysplastic syndromes are not seen initially, but may appear in the clinical course of the disease with progression to myelodysplastic syndrome (Erkurt et al., 2005). Furthermore, progression to aplastic anemia (Meytes et al., 1984; King et al., 1997) and association with hematologic malignancies (Geissler et al., 1987), as well as presence of concurrent anemia has been reported (Niparuck et al., 2008).

The exact underlying pathogenic mechanisms remain unknown to some extent, but successful treatment with immunosuppressive agents points towards an autoimmune-mediated process. The variable outcome with different treatments, however, is indicative of not only a singular mechanism of pathogenesis. Indeed, inhibition at the level of the thrombopoietin receptor (Hoffman et al., 1989) due to a humoral agent, antibodies against thrombopoietin itself (Katsumata et al., 2003) or a T-cell mediated process (Gewirtz et al., 1986; Doubek et al., 2006) have been described.

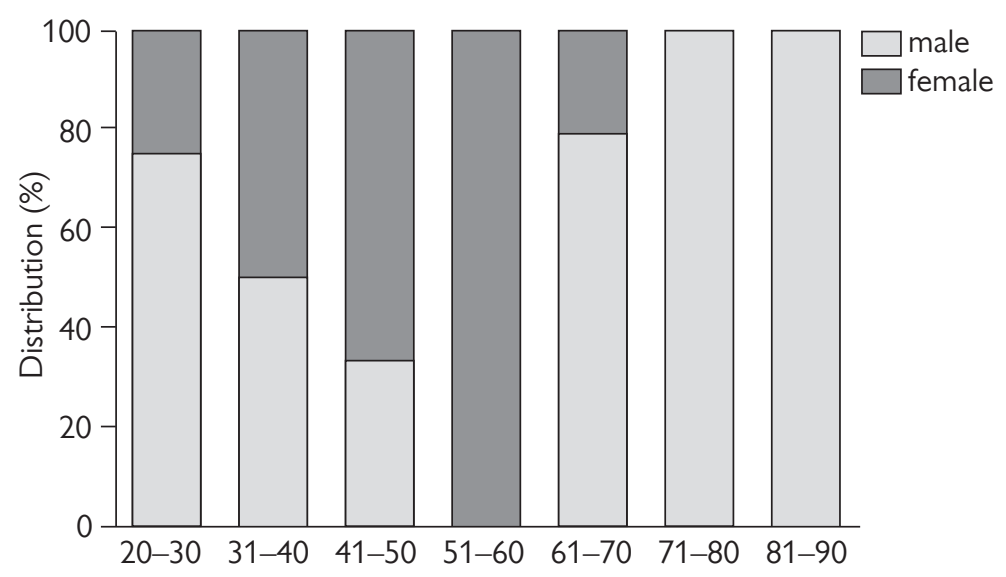

Figure 1 - Graphical representation of age and gender distribution of 30 patients with diagnosed AATP. 
In contrast to that, congenital amegakaryocytic thrombocytopenia (CAMT) is characterized by a mutation in the MPL gene coding for the TPO (thrombopoietin) receptor and is inherited in an autosomal recessive manner. Depending on the type of mutation, CAMT can be further classified into type-I or type-II according to the type of underlying mutation predicted to result in either complete loss of function or some retained function of the TPO receptor (nonsense and missense mutation, respectively) (King et al., 2005). However, CAMT without mutation in the MPL gene has been described in association with other diseases (e.g. Hoyeraal-Hreidarsson syndrome) and is referred to as type-III (King et al., 2004).

To date, allogeneic hematopoietic stem cell transplantation remains the only therapeutic option, whilst gene therapy or TPO agonists binding to partially functioning TPO receptors might provide benefit in the future.

Finally, AATP and CAMT have to be, amongst other things, distinguished from ITP, which is characterized by either an increased degradation of thrombocytes due to autoantibodies (Cines et al., 2009), inhibition of thrombocytopoiesis by autoantibodies interfering with megakaryopoiesis (Chang et al., 2003; McMillan et al., 2004), T-cell mediated toxicity towards thrombocytes (Olsson et al., 2003) or relatively insufficient thrombopoietin concentration (Emmons et al., 1996).

No primary therapy for AATP has been established to date. Immunosuppression, however, remains the mainstay of therapy. Treatments shown to be successful include the use of corticoids (Sakurai et al., 1984), intravenous immunglobulins (IVIG) (Leach et al., 1999), cyclosporine (Omri et al., 2010), anti-thymocyte globulin (ATG) (Niparuck et al., 2008), as well as allogeneic stem cell transplantation (Lonial et al., 1999) and anti-CD20 antibodies (Deeren and Dorpe, 2010; Mirzania et al., 2014). While these and other therapeutic agents have shown a wide range of response rates, reports emerged reporting success with thrombopoietin (TPO) receptor agonists recently; a treatment option that has already been theorized earlier (Lown et al., 2010). Published case reports include the use of Elthrombopaq in a patient with AATP and associated systemic lupus erythematosus (SLE) (Cela et al., 2010), as well as Romiplostim in a patient without underlying autoimmune disease (Shigekiyo et al., 2015).

\section{Material and Methods}

The literature review was performed with searches on google scholar using the keywords "acquired amegakryocytic", "acquired pure megakaryocytic aplasia". Statistical analysis was performed on 30 patients (including the patient from this report) published in 19 articles. For this, only publications and case reviews reporting adult patients without significant comorbidities and autoimmune disease have been included. Gender, age and treatment modality have been analysed using Excel. Patients receiving simultaneous treatment with more than one agent have been excluded from response rate analysis. 


\section{Case report}

A 61-year-old Caucasian male with a history of recent epistaxis, easy bruising and petechiae, which developed suddenly two weeks before admission. The patient history includes a perforated sigma diverticulitis 9 years ago, a total endoprothesis of the knee 2006, as well as a peripheral arterial occlusive disease stage Ila, adiposity with a BMI (body mass index) of 35 and a nicotine abuse of 45 pack years. No allergies or intolerances are known. On admission in a peripheral hospital due to severe epistaxis, blood workup showed marked thrombocytopenia of $0 / \mathrm{nl}$ and makrocytic, hyperchromic anemia (10.2 g/dl; Table 1), which worsened progressively with values reaching $7.4 \mathrm{~g} / \mathrm{dl}$ five days later, probably due to several episodes of intermittent epistaxis. EDTA (ethylenediaminetetraacetic acid) induced pseudothrombocytopenia was ruled out.

Serologically there was no evidence of a hepatitis B, C or HIV infection. Vitamin $B_{12}$ concentration was normal, folic acid was mildly reduced at $4 \mathrm{ng} / \mathrm{l}$ and substitution was started until concentration above the upper normal limit was detected. Autoimmune diagnostics showed normal lupus anticoagulans concentration as well as a normal lupus sensitive - apTT. No thrombocyte antibodies were detected, coombs test was negative. An ultrasound of the abdomen showed no splenomegaly and lactate dehydrogenase (LDH) was within normal limits.

Suspecting a case of immune thrombocytopenia the patient initially received a prednisone burst with $250 \mathrm{mg} /$ day and, due to refractory thrombocytopenia, subsequently $80 \mathrm{~g}$ intravenous immunoglobulins, which did not lead to a rise in

Table 1 - Initial and subsequent blood counts

\begin{tabular}{lcccc}
\hline & Initially & $\begin{array}{c}\text { Approx. } \\
\text { 1 month later }\end{array}$ & $\begin{array}{c}\text { Approx. } \\
\text { 14 months later }\end{array}$ & Unit \\
\hline Hemoglobin & 10.2 & 8.4 & $8.9 *$ & $\mathrm{~g} / \mathrm{dl}$ \\
Hematokrit & 30.0 & 0.24 & 0.25 & $\mathrm{I} / \mathrm{l}$ \\
Erythrocytes & 3.1 & 2.5 & 3.1 & $\mathrm{pl}$ \\
$\mathrm{MCH}$ & 33.0 & 34.0 & 29.0 & $\mathrm{gg}$ \\
$\mathrm{MCHC}$ & 34.0 & 35.0 & 36.0 & $\mathrm{gl}$ \\
$\mathrm{MCV}$ & 98.0 & 97.0 & 81.0 & $\mathrm{fl}$ \\
Leukocytes & 6.05 & 4.46 & 0.57 & $/ \mathrm{nl}$ \\
Thrombocytes & 0.0 & 8.0 & $22.0 *$ & $\%$ \\
Neutrophils & 64.0 & 59.2 & & $\%$ \\
Lymphocytes & 22.0 & 29.4 & & $\%$ \\
Monocytes & 9.3 & 7.5 & & $\%$ \\
Eosinophils & 4.5 & 1.3 & & $\%$ \\
Basophils & 0.2 & 0.0 & & $\%$ \\
\hline
\end{tabular}

*transfusions received; $\mathrm{MCH}$ - mean corpuscular hemoglobin; $\mathrm{MCHC}$ - mean corpuscular hemoglobin concentration; $\mathrm{MCV}$ - mean corpuscular volume 
thrombocyte counts. Due to symptomatic anemia the patient received several erythrocyte transfusions. He was then referred to our clinics for further diagnostics and treatment.

Upon admission we decided to try another cycle of corticoids (Dexamethasone $40 \mathrm{mg} /$ day, 4 days) and intravenous immunoglobulins at a concentration of $1 \mathrm{~g} / \mathrm{kg}$ body weight (BW) $(110 \mathrm{~g})$ to confirm the refractoriness to these immunosuppressive agents. We also performed bone marrow cytology, flow cytometry of bone marrow blood and a bone marrow biopsy with subsequent histological examination, as well as chromosomal analysis. The cytology showed a near to complete absence of megakaryocytes with preserved granulo- and erythropoiesis. Slight dysplastic changes such as minimal misshaped nuclei and pseudo-pelger cells could be seen upon cytological examination. Ring sideroblasts were not observed.

Repeated chromosomal analysis showed normal male karyotype $\mathrm{XY}, 46$. Flow-cytometry revealed no lead towards myelodysplasia and histological examination of the bone marrow biopsy confirmed the presence of normal maturation of the white and red lineage with nearly total absence of megakaryocytes as well as minimal increase in interstitial reticular fibers. There was no sign of an intramedullary increase in CD34+ cells and no infiltration by lymphoid cells. Erythropoietin concentration was well above normal limits.

During the in-hospital stay the patient developed fever with temperatures around $39{ }^{\circ} \mathrm{C}$, which was initially treated with Tazobactam/Piperacillin i.v. and then escalated to Meropenem i.v., and empirical Caspofungin i.v., as well as Clarithromycin p.o. after several days in a stepwise manner prior to further diagnostics due to suspicion of atypical pneumonia. Blood cultures showed no bacterial growth. In order to localize the focus of the suspected infection a CT (computed tomography) scan of the chest was performed which showed no pneumonia, but incidentally revealed an $2 \times 1.4 \mathrm{~cm}$ adrenal adenoma with partially negative Hounsfield units. The fever resolved during the course of treatment and

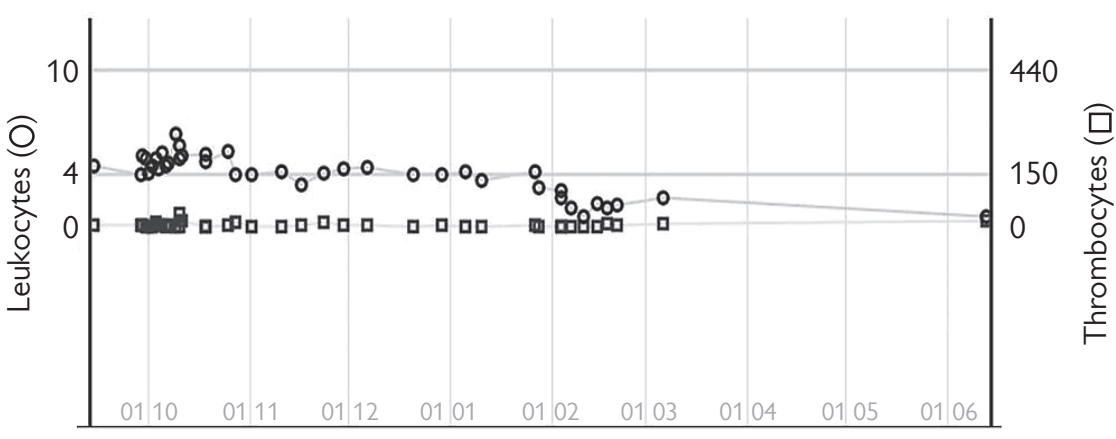

Figure 2 - Thrombocyte and leukocyte counts during therapy. 
C-reactive protein concentration decreased accordingly so that antibiotic and antimycotic treatment was discontinued.

In summary of the results we then diagnosed the patient with acquired amegakaryocytic thrombocytopenic purpura. Due to the risks associated with immunosuppressive agents such as Ciclosporine, or Cyclophosphamide we decided to initiate a therapy with $50 \mathrm{mg} /$ day Elthrombopaq, a TPO receptor agonist available for treatment of immune thrombocytopenia (ITP), which was successfully used in a patient with AATP and systemic lupus erythematosus (SLE) (Cela et al., 2010). After several days of treatment with Elthrombopaq we initially observed a rise in thrombocyte count and a decreased tendency to bleed. Thrombocyte counts, however, fell again after several weeks and the dose subsequently increased by 25 to $75 \mathrm{mg} /$ day. Laboratory follow-ups showed no improvement of the thrombocyte count after several weeks, necessitating another hospitalization and change of treatment to a combination therapy of Romiplostim and Ciclosporine, which again did not result in thrombocyte counts $>50 /$ nl (Figure 2). Repeated bone marrow histology then showed fatty degeneration, absent megakaryopoiesis and little granulo/erythropoiesis. In accordance to that, peripheral blood analysis revealed progressive leukopenia, as well as anemia, necessitating further erythrocyte transfusions. Finally, the diagnosis of aplastic anemia was made. Further workup showed inconspicuous JAK2-exon12 sequencing and cytomegalovirus infection was ruled out. Sequencing of ASXL1, CBL, DNMT3A, EZH2, RUNX, SF3B1, SRSF2, TET2, TP53, U2AF1, ZRSR2 did not reveal any mutations other than SNPs in TP53 and EZH2. BCOR, BCORL1, DNMT3A sequencing was uneventful.

With regards to the progressive leukopenia, as well as aplastic anemia further therapy with an anti-CD20 antibody was deemed unsuitable at that time point and work-up for allogeneic stem cell transplantation has been started.

\section{Discussion}

The distribution of AATP among gender is equal, but differs according to age. While most affected females are in the 40-60 age, more men are affected at both ends of age distribution (Figure 1) with a peak in the 60ties.

Analysis of published reports reveals a success rate of $<20 \%$ using either corticoids or IVIG alone. Treatment with Ciclosporine yielded a response rate of about $50 \%$ compared to $80 \%$ with anti-thymocyte globulin (ATG). Two cases were successfully treated with anti-CD20 antibodies, while there is only one case report of successful allogeneic stem cell transplantation in a patient refractory to multiple immunosuppressive agents and one report on successful use of Mycophenolate Mofetil (Bulchandani et al., 2007) (Table 2). In scarce reports, anti-CD20 antibodies and TPO receptor agonists showed $100 \%$ response ( $\mathrm{N}=2$ each).

With less than a $20 \%$ response rate, corticoids and IVIG are insufficient as first line therapy in AATP. Treatment with ATG seems promising, but carries the risk of allo-immunization and requires in-hospital stay. Ciclosporine on the other 


\section{Table 2 - Percent response to specific therapy}

\begin{tabular}{lcc}
\hline Treatment & Response rate $(\%)$ & $\mathrm{N}$ \\
\hline Corticoids & 9 & 22 \\
Intravenous immunoglobulin & 14 & 7 \\
Ciclosporine & 50 & 6 \\
Anti-thymocyte globulin & 80 & 5 \\
\hline
\end{tabular}

hand greatly increases the rate of infections, as well as the risk of liver damage necessitating close monitoring of liver enzymes and kidney function. Whether antiCD20 therapy represents a better therapeutic option remains to be assessed. Due to the serious side effects accompanied with an allogeneic stem cell transplantation, we feel that this treatment option should be reserved for those in certain age, refractory to more than one therapeutic option other than corticoids or IVIG and absence of significant comorbidities. TPO receptor agonists seem to be a reasonable first line therapy due to their mechanism of action even despite our negative result.

Our case report shows the importance of complete diagnostics in patients presenting with apparent immune thrombocytopenia and their regular follow-ups. It unrolls the lack of an optimal treatment algorithm for AATP and depicts the importance of case reports for this entity in order to assess treatment success with different therapeutic regimens, since prospective randomized clinical studies are difficult to perform due to the rarity of the disease.

\section{References}

Bulchandani, D., Nachnani, J., Belt, R., Hinton, S. (2007) Acquired pure megakaryocytic aplasia: Report of a single case treated with mycophenolate mofetil. Am. J. Hematol. 82, 650-651.

Cela, I., Miller, I. J., Katz, R. S., Rizman, A., Shammo, J. M. (2010) Successful treatment of amegakaryocytic thrombocytopenia with eltrombopag in a patient with systemic lupus erythematosus (SLE). Clin. Adv. Hematol. Oncol. 8, 806-809.

Chang, M., Nakagawa, P. A., Williams, S. A., Schwartz, M. R., Imfeld, K. L., Buzby, J. S., Nugent, D. J. (2003) Immune thrombocytopenic purpura (ITP) plasma and purified ITP monoclonal autoantibodies inhibit megakaryocytopoiesis in vitro. Blood 102, 887-895.

Cines, D. B., Bussel, J. B., Liebman, H. A., Luning Prak, E. T. (2009) The ITP syndrome: Pathogenic and clinical diversity. Blood 113, 6511-6521.

Deeren, D., Dorpe, J.V. (2010) Effective use of rituximab for acquired amegakaryocytic thrombocytopenia. Am. J. Hematol. 85, 977-978.

Doubek, M., Koristek, Z., Havranova, D., Smardova, L., Mayer, J. (2006) Megakaryocyte colony-forming unit growth is enhanced by alemtuzumab: in vitro experiments and a case report of acquired amegakaryocytic thrombocytopenic purpura. Leukemia 20,1618-1619.

Emmons, R. V., Reid, D. M., Cohen, R. L., Meng, G., Young, N. S., Dunbar, C. E., Shulman, N. R. (1996) Human thrombopoietin levels are high when thrombocytopenia is due to megakaryocyte deficiency and low when due to increased platelet destruction. Blood 87, 4068-4071. 
Erkurt, M. A., Kaya, E., Baran, M., Yitmen, E., Senel, S., Kuku, I., Aydogdu, I. (2005) Rapid progression of acquired amegakaryocytic thrombocytopenia to myelodysplastic syndrome: case report. Turk. J. Haematol. 22, 205-208.

Ernestho-ghoud, I. M., Rahamefy, O., Ranaivo, I. M., Andrianarison, M., Ramarozatovo, L. S., Rabenja, F. R. (2015) Acquired amegakaryocytic thrombocytopenia purpura: think of systemic lupus erythematosus. Pan Afr. Med.J. 20, 86. (in French)

Geissler, D., Thaler, J., Konwalinka, G., Peschel, C. (1987) Progressive preleukemia presenting amegakaryocytic thrombocytopenic purpura: Association of the 5q- syndrome with a decreased megakaryocytic colony formation and a defective production of Meg-CSF. Leuk. Res. 11, 731-737.

Gewirtz, A. M., Sacchetti, M. K., Bien, R., Barry, W. E. (1986) Cell-mediated suppression of megakaryocytopoiesis in acquired amegakaryocytic thrombocytopenic purpura. Blood 68, 619-626.

Hashimoto, A., Kanisawa, Y., Fujimi, A., Nakajima, C., Hayasaka, N., Yamada, S., Okuda, T., Minami, S., Yamauchi, N., Iwasaki, S., Suzuki, A., Kato, J. (2016) Thrombocytopenia and anemia with anti-c-Mpl antibodies effectively treated with cyclosporine in a patient with rheumatoid arthritis and chronic renal failure. Intern. Med. 55, 683-687.

Hoffman, R., Bruno, E., Elwell, J., Mazur, E., Gewirtz, A. M., Dekker, P., Denes, A. E. (1982) Acquired amegakaryocytic thrombocytopenic purpura: a syndrome of diverse etiologies. Blood $\mathbf{6 0}$, 1173-1178.

Hoffman, R., Briddell, R. A., van Besien, K., Srour, E. F., Guscar, T., Hudson, N.W., Ganser, A. (1989) Acquired cyclic amegakaryocytic thrombocytopenia associated with an immunoglobulin blocking the action of granulocyte-macrophage colony-stimulating factor. N. Engl. J. Med. 321, 97-102.

Katsumata, Y., Suzuki, T., Kuwana, M., Hattori, Y., Akizuki, S., Sugiura, H., Matsuoka, Y. (2003) Anti-c-Mpl (thrombopoietin receptor) autoantibody-induced amegakaryocytic thrombocytopenia in a patient with systemic sclerosis. Arthritis Rheum. 48, 1647-1651.

King, J. A., Elkhalifa, M.Y., Latour, L. F. (1997) Rapid progression of acquired amegakaryocytic thrombocytopenia to aplastic anemia. South. Med.J. 90, 91-94.

King, S., Germeshausen, M., Strauss, G., Welte, K., Ballmaier, M. (2004) Congenital amegakaryocytic thrombocytopenia (CAMT): A detailed clinical analysis of 21 cases reveals different types of CAMT. Blood 104, 740.

King, S., Germeshausen, M., Strauss, G., Welte, K., Ballmaier, M. (2005) Congenital amegakaryocytic thrombocytopenia: a retrospective clinical analysis of 20 patients. Br. J. Haematol. 131, 636-644.

Leach, J.W., Hussein, K. K., George, J. N. (1999) Acquired pure megakaryocytic aplasia report of two cases with long-term responses to antithymocyte globulin and cyclosporine. Am. J. Hematol. 62 , 115-117.

Lonial, S., Bilodeau, P. A., Langston, A. A., Lewis, C., Mossavi-Sai, S., Holden, J. T., Waller, E. K. (1999) Acquired amegakaryocytic thrombocytopenia treated with allogeneic BMT: a case report and review of the literature. Bone Marrow Transplant. 24, 1337-1341.

Lown, R., Rhodes, E., Bosworth, J., Shannon, M. S., Stasi, R. (2010) Acquired amegakaryocytic thrombocytopenia: potential role of thrombopoietin receptor agonists. Clin. Adv. Hematol. Oncol. 8, 809-812.

Lugassy, G. (1996) Non-Hodgkin's lymphoma presenting with amegakaryocytic thrombocytopenic purpura. Ann. Hematol. 73, 41-42.

McMillan, R.,Wang, L., Tomer, A., Nichol, J., Pistillo, J. (2004) Suppression of in vitro megakaryocyte production by antiplatelet autoantibodies from adult patients with chronic ITP. Blood 103, 1364-1369.

Meytes, D., Levi, M., Virag, I., Fried, D. (1984) Acquired amegakaryocytic thrombocytopenia with rapid progression into aplastic anemia. Harefuah 106, 509-510. (in Hebrew) 
Mirzania, M., Khalili, S., Hasanpoor, A., Shamshiri, A. R. (2014) Anti-CD20 antibody is effective in the patient with refractory amegakaryocytic thrombocytopenia, 25 months follow up. Int. J. Hematol. Oncol. Stem Cell Res. 8, 41-44.

Niparuck, P., Atichartakarn, V., Chuncharunee, S. (2008) Successful treatment of acquired amegakaryocytic thrombocytopenic purpura refractory to corticosteroids and intravenous immunoglobulin with antithymocyte globulin and cyclosporine. Int. J. Hematol. 88, 223-226.

Olsson, B., Andersson, P. O., Jernas, M., Jacobsson, S., Carlsson, B., Carlsson, L. M., Wadenvik, H. (2003) T-cell-mediated cytotoxicity toward platelets in chronic idiopathic thrombocytopenic purpura. Nat. Med. 9, 1123-1124.

Omri, H. E., Ibrahim, F., Taha, R. Y., Negm, R. H., Khinji, A. A., Yassin, M., Hijji, I. A., Ayoubi, H. E., Baden, H. (2010) Acquired pure megakaryocytic aplasia successfully treated with cyclosporine. Turk. J. Haematol. 27, 289-293.

Sakurai, T., Kono, I., Kabashima, T., Yamane, K., Nagasawa, T., Kashiwagi, H. (1984) Amegakaryocytic thrombocytopenia associated with systemic lupus erythematosus successfully treated by a high-dose prednisolone therapy.Jpn. J. Med. 23, 135-138.

Shigekiyo, T., Sekimoto, E., Shibata, H., Ozaki, S., Fujinaga, H., Hirose, T. (2015) Treatment of acquired amegakaryocytic thrombocytopenic purpura with romiplostim. Platelets 26, 504-506. 